\title{
Postoperative pancreatic fistula: a review of traditional and emerging concepts
}

This article was published in the following Dove Press journal:

Clinical and Experimental Gastroenterology

\section{Christopher B Nahm ${ }^{1-3}$ \\ Saxon J Connor ${ }^{4}$ \\ Jaswinder S Samra ${ }^{1,2,5}$ \\ Anubhav Mittal ${ }^{1,2,5}$}

'Upper Gastrointestinal Surgical Unit, Royal North Shore Hospital, Sydney, Australia; ${ }^{2}$ Northern Clinical School, Sydney Medical School, The University of Sydney, Sydney, Australia; ${ }^{3}$ Bill

Walsh Translational Cancer Research Laboratory, Kolling Institute, The

University of Sydney, Sydney, Australia: ${ }^{4}$ Department of Surgery, Christchurch Hospital, Christchurch, New Zealand; ${ }^{5}$ Australian Pancreatic Centre, Sydney, Australia
Correspondence: Anubhav Mittal Upper Gastrointestinal Surgical Unit, Level 8A, Acute Services Building, Royal North Shore Hospital, Reserve Road, St Leonards, Sydney, NSW 2065, Australia

Tel +6I 294632899

Email anubhav.mittal@sydney.edu.au

\begin{abstract}
Postoperative pancreatic fistula (POPF) remains the major cause of morbidity after pancreatic resection, affecting up to $41 \%$ of cases. With the recent development of a consensus definition of POPF, there has been a large number of reports examining various risk factors, prediction models, and mitigation strategies for this costly complication. Despite these strategies, the rates of POPF have not significantly diminished. Here, we review the literature and evidence regarding both traditional and emerging concepts in POPF prediction, prevention, and management. In particular, we review the evidence for the association between postoperative pancreatitis and POPF, and present a novel proposed mechanism for the development of POPF. Keywords: postoperative pancreatic fistula, postoperative pancreatitis, distal pancreatectomy, pancreaticoduodenectomy
\end{abstract}

\section{Introduction}

Postoperative pancreatic fistula (POPF) remains the main source of major morbidity and mortality after pancreatic resection, affecting between $13 \%$ and $41 \%$ of patients. ${ }^{1,2}$ POPF is associated with morbid sequelae including intra-abdominal sepsis and hemorrhage, carrying a mortality risk of $1 \%$ for all patients with POPF and $25 \%$ for patients with grade C POPF. ${ }^{3}$ The development of a POPF results in a complex and lengthy duration of inpatient care with a significant cost burden. Despite numerous reports and trials describing novel methods to curtail the risk of POPF formation, the reported rates of POPF have not significantly improved over the last three decades. ${ }^{4}$ This is largely attributable to the fact that the underlying mechanism of POPF is poorly understood, with only recent work beginning to reveal the role of postoperative pancreatitis (POP) in the development of POPF, rather than a mere loss of mechanical integrity of the pancreatoenteric anastomosis.

Early literature describing POPF has been heterogeneous due to varied definitions of POPF. The development of consensus definitions by the International Study Group on Pancreatic Fistula (ISGPF) ${ }^{5}$ has allowed for more uniformity in the reporting of this complication. These definitions have been further refined in 2016 to limit the reporting of POPF to only those that impact the postoperative clinical course of the patient. ${ }^{6}$ This has been crucial in allowing valid comparisons to be made between various interventions for the prevention and management of this complication.

The aim of this review is to discuss both traditional and emerging concepts in the understanding of POPF pathophysiology and management, with a focus on potential future directions for research in this field. 


\section{Definition}

Until 2005, there was no consensus in the definition and grading of POPF resulting in an inability to compare various interventions and preventive techniques for this complication. This led to great variability in the reported rate of POPF after pancreatic resection. An International Working Group of 37 pancreatic surgeons was formed in 2005 to establish for the first time a standardized all-inclusive definition of POPF to address this issue. This led to the first widely accepted definition of POPF: “drain output of any measurable volume of fluid on or after postoperative day 3 with an amylase content greater than 3 times the serum amylase activity."5 A grading system was also established which stratified patients from a relatively benign clinical course (grade A fistula), moderately unwell patients requiring medical or minimally invasive intervention (grade B), and critically ill patients, often with sepsis, requiring invasive intervention (grade C).

This consensus definition was revised in 2016 mainly to restrict the definition of POPF to only those that were "associated with a clinically relevant development/condition related directly to the postoperative pancreatic fistula" (ie, grade B and C). ${ }^{6}$ Thus, a grade A POPF has now been redefined and assigned the term "biochemical leak", as it does not cause any change to the clinical condition of the patient. The criteria for defining grade $\mathrm{B}$ and $\mathrm{C}$ POPFs were also made more specific to clarify the distinction between the two categories (Table 1). Importantly, this grading system has been repeatedly validated in terms of its association with other non-POPF complications, ${ }^{8}$ length of hospital and intensive care unit (ICU) stay, and the cost of hospital stay. ${ }^{9}$

\section{Traditional concepts Mechanism and risk factors}

The pathophysiology of POPF has been seldom evaluated and long assumed to be due to a gradual loss of mechanical integrity of the pancreatoenteric anastomosis leading to "leakage" of pancreatic fluid. There is a paucity of studies examining the precise mechanisms of POPF evolution, although many surgical methods to ameliorate this problem have been described, revealing a widespread misunderstanding of POPF as a mere loss of mechanical anastomotic integrity. Such reported methods emphasize mechanical buttressing or reinforcement of the anastomosis or staple line, including the use of fibrin sealants, ${ }^{10}$ autologous tissue patches, ${ }^{11}$ bioabsorbable mesh, ${ }^{12}$ varied suturing techniques, ${ }^{13}$ stents, ${ }^{14}$ and various methods of pancreatoenteric anastomosis. ${ }^{15}$ Very few of these have been consistently demonstrated in multiple high-volume randomized controlled trials (RCTs) to be effective in significantly reducing the rate or severity of POPF. ${ }^{16}$

Many cohort studies have examined and identified risk factors for the development of POPF. The factors most consistently shown to be predictive of POPF after pancreaticoduodenectomy (PD) include soft gland texture, non-pancreatic cancer non-chronic pancreatitis pathology, small pancreatic duct diameter ( $<3 \mathrm{~mm}$ ), and high intraoperative blood loss $(>1000 \mathrm{~mL})$. These features may be used to determine the Fistula Risk Score (FRS), which is a validated scoring system used to predict the risk of POPF formation after PD. ${ }^{17}$ Other reported but less-validated risk factors include that of increased body mass index (BMI), ${ }^{18}$ excess intraoperative fluid administration, ${ }^{19}$ increased pancreatic parenchymal remnant volume, ${ }^{20}$ poor preoperative nutrition, ${ }^{21}$ and male gender. ${ }^{22}$ The mechanisms by which such preoperative factors lead to POPF have not been studied in detail.

Although advanced age is associated with increased perioperative mortality, there seems not to be an increased risk of POPF. In a study comparing patients $<75(n=422)$ and $\geq 75$ $(n=102)$ years of age undergoing PD, the latter age category was associated with a higher rate of perioperative mortality ( $1.9 \%$ vs $5.9 \%, p=0.037)$, but not associated with increased risk of POPF $(10 \%$ vs $7.8 \%, p=0.579) .{ }^{23}$

Table I 2017 ISGPF definitions and grades of postoperative pancreatic fistula ${ }^{6}$

\begin{tabular}{|c|c|c|c|}
\hline Event & Biochemical leak & Grade B POPF & Grade C POPF \\
\hline Drain amylase concentration $>3 \times$ upper limit of normal serum value & Yes & Yes & Yes \\
\hline Persisting peripancreatic drainage $>3$ weeks & No & Yes & Yes \\
\hline Clinically relevant change in the management of POPF & No & Yes & Yes \\
\hline Percutaneous or endoscopic drainage of POPF-associated collections & No & Yes & Yes \\
\hline Angiographic procedures for POPF-associated bleeding & No & Yes & Yes \\
\hline Reoperation for POPF & No & No & Yes \\
\hline Signs of infection related to POPF & No & $\begin{array}{l}\text { Yes (without organ } \\
\text { failure) }\end{array}$ & $\begin{array}{l}\text { Yes (with organ } \\
\text { failure) }\end{array}$ \\
\hline POPF-related organ failure & No & No & Yes \\
\hline POPF-related death & No & No & Yes \\
\hline
\end{tabular}

Abbreviations: ISGPF, International Study Group on Pancreatic Fistula; POPF, postoperative pancreatic fistula. 


\section{Sequelae of POPF}

The sequelae of POPF can be broadly divided into two specific categories: hemorrhage and sepsis. Either of these may further lead to other systemic consequences including prolonged hospital stay, delayed gastric emptying (DGE), enteric fistulae, multiorgan failure, and/or death.

\section{Hemorrhage}

Hemorrhagic complications are highly feared sequela of POPF due to their ability to cause dramatic and rapid deterioration of the patient. Most commonly, this occurs due to a pseudoaneurysm of a large visceral artery that develops due to prolonged contact with pancreatic fluid which has a high proteolytic capacity. ${ }^{24}$ Arteries that are commonly involved include the common hepatic, splenic, gastroduodenal, and superior mesenteric artery. Such pseudoaneurysms also may be discovered at the time of postoperative axial imaging performed for other reasons, and if left untreated, may increase in size leading to rupture, which is usually associated with severe hemorrhage and hemodynamic instability. Therefore, early treatment of these lesions is of paramount importance, and will be discussed in further detail below (see "Management" section).

\section{Sepsis}

Infective sequelae of pancreatic resection are more common after PD than after distal pancreatectomy (DP), presumably due to contamination of the operative field by biliary and enteric content during the anastomotic phase of the operation. The development of this complication is often insidious and manifests clinically as the development of fever, DGE, abdominal pain, and/or rising inflammatory markers. Turbidity of drain fluid may also herald intra-abdominal sepsis, and should be evaluated with Gram stain, microscopy, and culture. Contrast-enhanced CT imaging should be performed to evaluate such patients to determine whether there are any drainable fluid collections. On CT imaging, a walled-off peripancreatic fluid collection may be seen representing an abscess. Such collections are most commonly managed with minimally invasive radiologically guided percutaneous or endoscopic drainage and rarely require laparotomy.

\section{Related major complications}

Patients who develop POPF are also more likely to develop non-fistula postoperative complications including prolonged duration of hospital stay, wound infection, acute cardiac events, bile leak, and mortality. ${ }^{25}$ Notably, POPF and POP have both been identified as predictors of the development of DGE after PD, which leads to poorer nutritional outcomes and increased length of hospital stay. ${ }^{26}$

\section{Prediction of POPF}

Early prediction of the development of a pancreatic fistula is important as it allows for identification of at risk patients who will need close monitoring, and equally the implementation of enhanced recovery protocols in patients who are deemed to be at low risk. ${ }^{27}$ In addition, the early diagnosis of POPF allows prompt intervention to prevent the development of disastrous and potentially lethal sequelae.

\section{Preoperative}

The type of resection planned for the patient is known to influence the rate of POPF formation. POPF rates for PD, DP, and central pancreatectomy $(\mathrm{CP})$ are $13 \%,{ }^{2} 29 \%,{ }^{28}$ and $41 \%,{ }^{1}$ respectively. Rates of POPF after enucleation of pancreatic tumors have been reported to be as high as $73 \%$, with the risk particularly high when the tumor is located $<3 \mathrm{~mm}$ from the main pancreatic duct. ${ }^{29}$ The high rates of POPF after CP occur due to the existence of both a remnant pancreatic stump and a pancreatoenteric anastomosis. $\mathrm{CP}$ and enucleation are usually only justified in healthy and young individuals where the lesion is benign, where any other resection (eg, subtotal pancreatectomy) is likely to cause severe long-term morbidity (eg, pancreatic exocrine insufficiency, diabetes mellitus) in an otherwise fit patient. ${ }^{30}$

Rates of POPF are lowest for patients who have a histopathological diagnosis of either pancreatic ductal adenocarcinoma (PDAC) or chronic pancreatitis, as both are associated with atrophy of the remnant gland due to chronic ductal obstruction. ${ }^{17}$ This results in a fibrotic pancreas with a widened pancreatic duct in which a pancreatoenteric anastomosis may be technically easier to achieve.

Some authors have reported the use of preoperative axial imaging studies to predict POPF formation by characterizing its enhancement patterns. Preoperative imaging can provide some insight into important factors such as the consistency of the future remnant pancreas and also the presence of pancreatic ductal dilatation. In a cohort of 29 patients undergoing pancreatic resection, magnetic resonance imaging findings of a higher pancreas-to-muscle signal intensity ratio on $\mathrm{T} 1$ images were associated with a higher risk of POPF. A signal intensity ratio cutoff value of 1.41 had a positive predictive value of $73 \%$ and a negative predictive value of $89 \%$ with regard to the development of POPF. ${ }^{31}$ In another study of 146 patients undergoing PD using triple phase CT imaging, a slower rate of contrast enhancement of the remnant gland 
was associated with a lower rate of POPF formation. ${ }^{32}$ This likely relates to a more fibrotic and less vascular pancreatic remnant. Such reports have been observed in small observational cohorts with results yet to be validated in larger sample sizes.

\section{Intraoperative}

The FRS is the most widely used predictive tool, utilizing several intraoperatively determined factors that have repeatedly been shown to predict the development of POPF during a PD. This scoring system (Tables 2 and 3) utilizes gland texture, histopathological diagnosis, pancreatic duct diameter, and intraoperative blood loss to assign a score out of 10 , which is then used to determine whether the patient is at negligible (score 0 ), low (score 1-2), moderate (score $3-6$ ), or high (score 7-10) risk of developing a POPF. ${ }^{17}$ In a multi-institutional validation study of the FRS evaluating 594 patients who underwent $\mathrm{PD}$, the incidence of grade $\mathrm{B} / \mathrm{C}$ POPF was $6.6 \%$ for low risk, $12.9 \%$ for moderate risk, and $28.1 \%$ for high-risk patients. ${ }^{33}$

Table 2 Fistula Risk Scoring system for the prediction of postoperative pancreatic fistula ${ }^{17}$

\begin{tabular}{lll}
\hline Risk factor & Parameter & Points* \\
\hline Gland texture & Firm & 0 \\
Pathology & Soft & 2 \\
& PDAC or chronic & 0 \\
& Pancreatitis & \\
& Ampullary, duodenal, & 1 \\
Pancreatic duct & cystic, islet cell, etc & \\
diameter & $\geq 5 \mathrm{~mm}$ & 0 \\
& $4 \mathrm{~mm}$ & 1 \\
& $3 \mathrm{~mm}$ & 2 \\
& $2 \mathrm{~mm}$ & 3 \\
Intraoperative & $\leq 1 \mathrm{~mm}$ & 4 \\
blood loss & $\leq 400 \mathrm{~mL}$ & 0 \\
& $40 \mathrm{I}-700 \mathrm{~mL}$ & 1 \\
& $70 \mathrm{I}-1000 \mathrm{~mL}$ & 2 \\
& $>1000 \mathrm{~mL}$ & 3 \\
\hline
\end{tabular}

Note: *Out of 10.

Abbreviation: PDAC, pancreatic ductal adenocarcinoma.

Table 3 FRS zones and probability of POPF after PD - results from a multi-institutional validation study of 594 PD patients ${ }^{33}$

\begin{tabular}{lll}
\hline FRS points (out of I0) & Risk zone & Risk of POPF* (\%) \\
\hline 0 & Negligible & $* *$ \\
I-2 & Low & 6.6 \\
$3-6$ & Moderate & 12.9 \\
$7-10$ & High & 28.1 \\
\hline
\end{tabular}

Notes: ${ }^{*}$ Clinically relevant. ${ }^{*}$ No patients in this validation cohort were of negligible risk.

Abbreviations: FRS, Fistula Risk Score; PD, pancreaticoduodenectomy; POPF, postoperative pancreatic fistula.

\section{Postoperative}

Both macroscopic and biochemical analyses of drain effluent are significant predictors of POPF development. Macroscopically, drain fluid that is red-brown in colour in the first few postoperative days is thought to be associated with enzymatic breakdown of intra-abdominal proteins caused by a leakage of protease-rich pancreatic fluid. ${ }^{5}$ Biochemically, amylase concentration in the drain fluid in the postoperative phase is a strong predictor of POPF development. In a prospective validation study of patients undergoing $\mathrm{PD}$, a postoperative day 1 drain amylase level of $<600 \mathrm{U} / \mathrm{L}$ was found to be a stronger predictor of the absence of POPF than a soft gland and duct diameter. ${ }^{34}$

\section{Prevention of POPF}

\section{Nutrition}

A number of studies have demonstrated both obesity and malnutrition as risk factors for the development of POPF. In a prospective cohort study of 87 patients undergoing PD, a higher BMI and lower prognostic nutritional index were significantly associated with a higher risk of POPF. ${ }^{35}$ In addition, rates of POPF have been found to be higher in patients with sarcopenia. ${ }^{36}$ Although preoperative nutritional optimization has not been evaluated in randomized trials for its capacity to prevent POPF, multimodal prehabilitation prior to major oncological surgery has been shown to decrease postoperative morbidity. ${ }^{37}$ Units performing pancreatectomy should therefore consider the involvement of specialist dieticians in the preoperative phase to screen patients for malnutrition and for the purposes of prehabilitation.

In the postoperative phase, the optimal route of the administration of nutrition has yet to be definitively confirmed. A systematic review of seven RCTs and eight retrospective cohort studies comparing enteral and total parenteral nutrition after PD did not show a significant difference in rates of POPF with any particular feeding modality. ${ }^{38} \mathrm{~A}$ number of RCTs exploring these two modalities also do not demonstrate a significant difference. ${ }^{39-41}$ One retrospective cohort study demonstrated a $6 \%$ re-laparotomy rate for tube-related complications in patients who received a feeding jejunostomy at the time of operation. ${ }^{42}$ The use of "immunonutrition" via arginine- and omega-3-enriched enteral feeds has been demonstrated to reduce postoperative complications and length of hospital stay in one RCT. ${ }^{41}$

One multicenter RCT comparing early enteral and total parental nutrition in patients undergoing PD demonstrated an increase in overall postoperative complications and rate and severity of POPF in patients receiving early enteral feeds 
via the nasojejunal route. ${ }^{43}$ Notably, the increase in rate of enteral feeds in this study was in $25 \mathrm{~mL} /$ hour increments per day, which is considerably faster than standard rates. ${ }^{44}$ Whether such an increase in rates of complication persists at a slower rate of enteral feed infusion needs to be explored in further prospective studies.

\section{Neoadjuvant chemotherapy (NAC)/radiotherapy}

The role of NAC and/or radiotherapy is a contentious issue and its oncological benefit has yet to be demonstrated in the setting of large volume RCTs, but is becoming more widely utilized in the setting of pancreatic cancer. ${ }^{45}$ This has led to a number of cohort studies evaluating the effect of NAC on postoperative morbidity, particularly with regard to the development of POPF. In a review of American College of Surgeons National Surgical Quality Improvement Program (ACS-NSQIP) data, postoperative outcomes from 3408 patients who underwent pancreatectomy were analyzed. In these data, neoadjuvant treatment was associated with lower rates of POPF $(10.2 \%$ vs $13.2 \%, p=0.017)$ despite higher intra/postoperative blood transfusion requirements (27.4\% vs $20.3 \%, p<0.0001)$. A retrospective analysis of 79 patients who underwent neoadjuvant chemoradiation prior to $\mathrm{PD}$ for periampullary malignancies demonstrated no difference in mortality, less POPF, and less intra-abdominal abscess compared with historical controls. ${ }^{46}$ These findings were replicated in another retrospective cohort study of 58 patients with PDAC who underwent DP, where 28 patients had received neoadjuvant chemoradiation, and 30 patients had upfront resection. The patients who received neoadjuvant chemoradiation experienced significantly less POPF, and lower drain amylase levels on postoperative day 1 and $3 .{ }^{47}$ Furthermore, NAC and radiotherapy alone have each been reported to be of benefit in reducing rates of POPF. ${ }^{48,49}$ The mechanisms by which neoadjuvant therapy reduces POPF rates are uncertain; however, it may in part relate to a longer period of pancreatic ductal obstruction and atrophy of the remnant gland, particularly in head of pancreas tumors.

\section{Pharmacological}

The use of somatostatin analogs remains a point of debate among many pancreatic surgeons, with significant heterogeneity in the literature with regard to its purported benefits and detriments despite the existence of $>10$ RCTs exploring this issue. ${ }^{50-60}$ Octreotide has long been used in pancreatic surgery due to its mechanism of action, which is to bind to G-coupled somatostatin receptors, thus exerting an inhibitory action on both exocrine and endocrine functions of the pancreas. ${ }^{61}$ Reduction in pancreatic exocrine secretions may encourage healing of pancreatoenteric anastomoses or staple lines. The benefit in reducing POPF and postoperative complications was demonstrated in a number of early randomized trials. In a multicenter trial of 246 patients undergoing elective pancreatic resection, the administration of octreotide was associated with a significantly lower rate of POPF, and also a lower rate of complications in those deemed to be at high fistula risk. ${ }^{51}$ These findings were supported by another multicenter RCT of 218 patients, which demonstrated a significantly lower rate of POPF in patients who received octreotide; however, it was not clear as to what proportions of these were clinically relevant. $^{62}$

These trials, however, have been criticized for an overall high rate of $\mathrm{POPF}$, and investigators have sought to determine the role of octreotide in a more modern surgical context. Lowy et al performed a single-institution RCT in 120 patients undergoing PD for periampullary malignancy. They found that the administration of octreotide was not associated with any significant reduction in POPF rates. ${ }^{63}$ These findings were supported by another RCT of 383 patients where no significant reduction in POPF rates was seen in the octreotide group. ${ }^{60}$ More recently, pasireotide, a longer acting somatostatin ana$\log$, has been evaluated in an RCT of 300 patients undergoing pancreatic resection. Whilst this has demonstrated a reduction in POPF rates, this drug has not been approved for use by the Food and Drug Administration in pancreatic surgery. ${ }^{64}$ Notably, in a recent large multinational retrospective cohort study with propensity score matched analysis, the use of octreotide was actually found to be associated with a greater risk of POPF development in patients with high-risk pancreata $(p<0.001) .{ }^{65}$ The use of octreotide, therefore, remains heterogeneous among pancreatic units across the globe.

\section{Intravenous fluids}

The role of goal-directed intravenous fluids in the perioperative phase is integral to care of the post-pancreatectomy patient. A number of cohort studies have shown an association with excess fluids and a higher risk of POPF formation. ${ }^{19,66} \mathrm{~A}$ moderately restrictive fluid regimen using hypertonic saline has also been demonstrated in a prospective randomized trial to be of benefit in the prevention of POPF and reduction of return to theater, readmissions, length of stay, and mortality. ${ }^{67}$ Whilst the benefit of hypertonic solutions requires validation in further prospective studies, a policy of goal-directed fluid administration appears to be a prudent measure. 


\section{Anastomosis}

The operative management of the remnant pancreas after partial pancreatic resection remains the topic of significant debate, with no clear optimal surgical technique having been demonstrated to decrease the rates of POPF. Consequently, there is significant variation in operative technique among high-volume pancreatic surgeons.

A number of pancreatoenteric anastomotic techniques have been evaluated in prospective studies in regard to the rate of POPF. This includes the comparison of pancreatogastrostomy (PG) with the more traditional pancreato-jejunostomy (PJ). PG is theoretically thought to reduce the rate of POPF by deactivating pancreatic enzymes in an acidic environment and the lack of trypsin activating enterokinase in the gastric epithelium. In the largest multicenter RCT to date of PG versus PJ during PD (RECOPANC study), 440 patients were randomized to one of these two anastomoses, revealing no significant difference in the rate of POPF ( $20 \%$ vs $22 \%$, $p=0.617$ ). However, $\mathrm{PG}$ was associated with an increased rate of grade $\mathrm{A} / \mathrm{B}$ post-pancreatectomy hemorrhage, perioperative stroke, reduced need for long-term exocrine enzymatic replacement, and some improvement in quality of life measures. A nonsignificant trend toward increase in the rate of POPF was observed when the surgery was performed by surgeons with an annual caseload of $<10$ per year versus $>25$ per year (OR 2.801, 95\% CI 1.155-6.794, $p=0.064$ ) ${ }^{68}$ A meta-analysis of eight RCTs comparing 607 patients who underwent $P G$ and 604 patients who underwent $P J$ revealed a significant reduction in POPF with the PG technique. ${ }^{69}$ However, a recent retrospective cohort study of 58 patients undergoing CP has demonstrated a higher rate of POPF in patients receiving $\mathrm{PG}$ as opposed to $\mathrm{PJ}^{70}$ The authors hypothesize that this may be due to a greater amount of tension that exists in a PG anastomosis as opposed to PJ.

Variations in PJ technique have also been explored, with the two most common being duct-to-mucosa and the invagination technique. Two recent RCTs comparing duct-tomucosa versus invagination pancreaticojejunostomy reveal no significant difference in the rate of POPF between the two techniques. ${ }^{71,72}$ However, one of the RCTs demonstrated a significantly lower POPF rate with the invagination technique in a subgroup analysis of patients with a soft pancreas $(10 \%$ vs $42 \%, p=0.010) .{ }^{71} \mathrm{~A}$ number of meta-analyses examining RCTs comparing these two techniques have been conducted, each showing no difference in the incidence of POPF. However, a significant degree of heterogeneity in the reported definition of POPF complicates the comparison between duct-to-mucosa and the invagination $\mathrm{PJ}$ technique. ${ }^{73-76}$
One retrospective study of 44 patients undergoing stapled closure of the pancreatic stump after PD reported a surprisingly low POPF rate of $13.6 \%$. This led the authors to suggest that avoidance of a pancreatoenteric anastomosis circumvents enterokinase-mediated activation of pancreatic enzymes, which in turn may explain the low POPF rate. ${ }^{77}$ Indications for stump closure in this series included a small main pancreatic duct diameter, soft texture of the pancreatic remnant, and personal experience of the surgeon. Evaluation of this technique in a randomized controlled trial setting has yet to be performed.

\section{Glues}

The use of fibrin sealants and acrylic glues to decrease the risk of POPF has also been investigated, but none have definitively demonstrated a clinical benefit. A prospective cohort study evaluating pancreatic ductal occlusion with acrylic glue (without pancreatoenteric anastomosis) versus conventional pancreaticojejunostomy after PD revealed no significant difference in clinically relevant POPF rates (OR 1.45, 95\% CI 0.012-1.096, $p=1.004) .^{78}$ A RCT comparing pancreatoenteric anastomosis with and without occlusion of the main pancreatic duct with fibrin glue similarly revealed no statistically significant difference in the rates of POPF $(15 \%$ vs $17 \%$, respectively), deep collections, or intra-abdominal collections. ${ }^{79}$ A meta-analysis of seven RCTs evaluating the use of fibrin sealants after partial pancreatectomy also revealed no significant difference in the rates of POPF (OR $0.83,95 \%$ CI $0.6-1.14, p=0.245$ ), although a trend toward less postoperative hemorrhage was seen with fibrin sealants (OR 0.43, 95\% CI 0.18-1.0, $p=0.05){ }^{80}$

\section{Stents}

The use of pancreatic stents has also been evaluated in the literature. In the context of PD, stents may be categorized as either internal or external. Internal stents are introduced into the main pancreatic duct, over which the pancreatoenteric anastomosis is fashioned. Conceptually, this is designed to divert pancreatic juice further downstream, away from the pancreatic anastomosis, and also to avoid inadvertent sutured closure of the pancreatic duct. Such stents have proven problematic, mainly with the issue of distal migration of the stent. Internal stents also seem not to significantly reduce the rates of POPF, with some studies demonstrating an increase in the rate of this complication especially in high-risk pancreata. ${ }^{65,81,82}$

Stents may also be externalized through the abdominal wall to drain extracorporeally. Several RCTs and one retrospective cohort study with propensity score matched 
analysis have reported a reduction in POPF rates with the use of external stents. ${ }^{65,81,83-85}$ However, a Cochrane review evaluating three RCTs comparing internal versus external stents failed to show superiority of one form of stent over the other in terms of POPF reduction. ${ }^{86}$ External stents have been associated with a significantly longer length of hospital stay than internal stents, likely due to complications of the outpatient management of such a prosthesis. ${ }^{87} \mathrm{~A}$ recent propensity score matched retrospective cohort analysis by Ecker et al of 522 patients with high-risk pancreata (FRS criteria) demonstrated a reduction in POPF rates with the use of externalized stents, and an increase in POPF rates with internal stents compared with no stents (external 15.2\% vs internal $43.8 \%$ vs no stent $33.8 \%, p<0.001){ }^{65}$

Transpapillary stents inserted by endoscopic retrograde cholangiopancreatography (ERCP) have been explored as a measure to prevent POPF after DP. A meta-analysis of one RCT and three cohort studies encompassing 200 patients demonstrated a significantly lower rate of POPF in patients who underwent preoperative transpapillary stenting. However, the results of the single RCT (showing an increase in the rate of POPF) rather demonstrated an increase in POPF rates. Given the risk of ERCP-induced pancreatitis in the setting and the lack of convincing evidence to support its routine use, it seems prudent to avoid transpapillary intervention where possible.

\section{DP stump}

The management of the remnant pancreatic stump after DP remains a significant challenge despite numerous methods described in an attempt to reduce the risk of POPF. Such methods include comparisons between stapled closure and the role of oversewing the pancreatic stump. A multicenter RCT in 450 patients comparing hand sewn versus stapled closure of the DP stump demonstrated no difference in POPF rates between the two techniques. ${ }^{88}$

One retrospective cohort study has shown that dedicated oversewing of the main pancreatic duct reduces the rates of POPF $(9.6 \%$ vs $34.0 \%, p<0.001) .{ }^{89}$ It is possible, however, that such a result was attained due to more accurate identification of the pancreatic duct in harder and more fibrotic pancreata, which is associated with a lower risk of POPF.

The application of a jejunal seromuscular patch to the DP staple line has also been investigated, with no change in the rates of POPF. ${ }^{90}$ Drainage of the remnant stump with a pancreatoenteric anastomosis has also been evaluated, with no significant difference in POPF rates demonstrated. ${ }^{91}$
A variety of ancillary methods have been described to reinforce stump closure of the remnant pancreas after DP. This includes the use of staple line reinforcement with an absorbable mesh. A number of small sample size prospective trials and retrospective cohort studies have demonstrated some reduction in POPF with its use. ${ }^{12,92,93}$ However, a multicenter RCT of 275 patients evaluating the use of an absorbable fibrin sealant patch (TachoSil) revealed no significant reduction in grade $\mathrm{B}$ or $\mathrm{C}$ POPF with its use patients undergoing DP (TachoSil 8\% vs no TachoSil 14\%, $p=0.139$ ). ${ }^{94}$ Various glues have also been evaluated, with most studies showing no reduction in the rates of POPF. Such glues include cyanoacrylate formulations ${ }^{95}$ and fibrin glues. ${ }^{10}$

\section{Drains}

Whether use of surgical intra-abdominal drains influences the risk of POPF and postoperative complications remains uncertain and a point of some contention. Conlon et $\mathrm{al}^{96}$ conducted an RCT of 179 patients who underwent pancreatic resection, randomized to having a drain or no drain placed at the time of surgery. In this study, patients with a drain had a higher incidence of POPF, intra-abdominal abscess and/or collection. However, another RCT by Van Buren et $\mathrm{al}^{97}$ of 137 patients undergoing PD demonstrated an increase in POPF, gastroparesis, intra-abdominal collection/abscess, need for a percutaneous drain, and length of hospital stay. Most recently, the 2016 PANDRA trial ${ }^{98}$ evaluated 395 patients undergoing $\mathrm{PD}$ randomized to either receiving an intra-abdominal drain or not at the time of surgery. This demonstrated a significant reduction in POPF rate $(5.9 \%$ vs $11.9 \%, p=0.030)$ and fistula-associated complications ( $13.0 \%$ vs $26.4 \%, p=0.0008)$ in patients who did not receive a surgical drain. The results unfortunately were not stratified by FRS in this cohort. Interestingly, there was a significantly larger proportion of patients who had undergone neoadjuvant therapy and received somatostatin analogs in the "no drain" group. Moreover, the use of drains was not seen to be independently associated with the need for reintervention on multivariable analysis. It currently seems appropriate to undertake a selective approach to drain placement, where avoidance of a drain may be suitable in those patients deemed to be at low risk of POPF (large pancreatic duct diameter and hard glandular consistency).

Patients who do receive an intra-abdominal drain may benefit from its early removal by reducing the risk of secondary infection. In a prospective study of 114 patients randomized to early (day 3 ) or late (day 5+) drain removal, the early removal group experienced reduced rates of abdominal 
complications and reduced pulmonary complications. However, patients with a drain amylase concentration of $>5000$ on postoperative day 1 were excluded from randomization. ${ }^{99}$ Moreover, there was a greater number of patients with smaller duct diameter in the late drain removal group, which may confound these results. Importantly, Penrose drains were used in this study, as opposed to the more popular closed drainage systems. The open nature of the former would theoretically make them more likely to be associated with infection. Thus, these data should be interpreted with caution. It may be appropriate to remove drains early in patients who are at a low risk of development of fistula (eg, with a low FRS).

\section{Minimally invasive pancreatectomy DP}

The use of a minimally invasive approach seems not to affect the rate of POPF in DP. In a propensity score matched analysis of laparoscopic $(n=51)$ versus open $(n=51)$ DP for pancreatic cancer, there was no significant difference in the rate of POPF ( $10 \%$ vs $19 \%$, respectively, $p>0.05) .{ }^{100}$ This is consistent with findings from another retrospective analysis of laparoscopic $(n=33)$ versus open $(n=46)$ DP for pancreatic cancer (POPF rates $9 \%$ vs $15 \%$, respectively, $p=0.42) .{ }^{101}$ In an analysis of ACS-NSQIP data for 1815 patients who underwent open $(n=921)$, laparoscopic $(n=694)$, or robotic $(n=200) D P$, no difference was seen in the rates of POPF (17\% vs $18 \%$ vs $21 \%$, respectively, $p=0.6484) .{ }^{102}$

\section{PD}

In a multi-institutional study with propensity score matched analysis of patients undergoing open $(n=152)$ versus robotic $(n=152) P D$, there was no significant difference seen in the rate of POPF (6.6\% vs $11.2 \%$, respectively, $p=0.23) .{ }^{103}$ Moreover, in a matched case-control study comparing postoperative outcomes of open $(n=93)$ versus laparoscopic $(n=93) P D$, there was no difference in the rates of POPF between these groups $(6.5 \%$ vs $6.5 \%, p=1.00) .{ }^{104}$ It must be emphasized that both these studies involved institutions performing a high volume of minimally invasive pancreatic resections.

\section{Management}

The key to management of POPF includes early recognition of this problem and also prevention of further life threatening sequelae such as bleeding and sepsis.

\section{Biochemical leak}

Patients with a biochemical leak of asymptomatic amylaserich drain effluent may be safely managed conservatively, but require multidisciplinary involvement to prevent its progression to a clinically relevant POPF.

\section{Observation}

Vigilant observation in this context involves close monitoring for signs of sepsis including fevers and/or rising inflammatory markers. These findings may herald the development of an infected intra-abdominal fluid collection. A contrastenhanced CT of the abdomen is usually required to assess for drainable collections and the commencement of broad spectrum antibiotic therapy.

\section{Nutrition}

From the perspective of nutrition, enteral rather than parenteral nutrition has been demonstrated in an RCT of 78 patients with POPF to be superior in spontaneous fistula closure rates. ${ }^{105}$ Somatostatin analogs were not administered in this particular trial. In situations where the pancreatoenteric anastomosis is isolated from the alimentary tract, it would be appropriate to recommence an oral diet as usual. In situations where such isolation does not exist (eg, PG), it may be advisable to perform an oral contrast study to ensure the absence of a mechanical anastomotic leak prior to resuming an oral diet.

\section{Somatostatin analogs}

There has been no clear benefit demonstrated in the use of somatostatin analogs to treat established POPF. Despite this, the use of this drug remains popular, possibly as a result of a number of early single armed case series reporting a high rate of spontaneous pancreatic fistula closure. ${ }^{106,107}$ A metaanalysis of seven RCTs evaluating a variety of somatostatin analogs (somatostatin, lanreotide, and octreotide) in 102 patients with established POPF showed that rates of POPF resolution were not increased with the use of these drugs. Due to significant inter-study heterogeneity, a comparison of POPF severity could not be performed. ${ }^{108}$

\section{Grade B/C pancreatic fistula}

Where the patient remains clinically stable, a "step-up" approach to POPF management is usually suitable. ${ }^{109}$

\section{Minimally invasive drainage techniques}

The evolution of interventional radiology has dramatically reduced the need for unplanned re-laparotomy after pancreatic resection in patients with POPF. Where a CT scan has detected an intra-abdominal peripancreatic collection that is safely accessible by percutaneous route, radiologically 
guided drainage of such collections has been demonstrated to be both effective and safe. ${ }^{10,111}$ In patients where the fluid collection is positioned in the lesser sac and cannot be reached by percutaneous route, endoscopic ultrasound may be utilized to drain these collections through the posterior wall of the stomach. ${ }^{112}$

Peripancreatic collections after DP are more likely to be sterile than after PD as there has been no breach of the bowel wall. Thus, a more conservative approach may be undertaken with these fluid collections occurring after DP, particularly where there is a low-risk pancreatic remnant. One observational study demonstrated that such collections occur in $43 \%$ of patients after DP, with over $90 \%$ spontaneously decreasing in size without further intervention. ${ }^{113}$

\section{Angiographic intervention}

In the setting of a patient with a grade B/C POPF, a significant drop in hemoglobin coupled with the development of hemodynamic instability should raise suspicion of an intraabdominal bleed. Such patients should be urgently evaluated with a CT angiogram to identify any arterial pseudoaneurysms and/or points of arterial hemorrhage, which may subsequently be managed with angiographic stenting and/or embolization. ${ }^{114,115}$ This minimally invasive approach to the management of such vascular complications is valuable as it often avoids the need for re-laparotomy and its associated risks and morbidity. Moreover, in a post-pancreatectomy patient, the development of postoperative adhesions can make difficult the task of rapidly identifying bleeding retroperitoneal vessels and achieving effective hemostasis.

\section{Emergency laparotomy}

Advances in interventional radiology and angiographic techniques have fortunately led to a decrease in the need for re-laparotomy for POPF. ${ }^{116}$ However, there are still circumstances in which open surgery is a more favorable option. These include situations in which there is catastrophic hemorrhage that requires evacuation of a large amount of blood and hematoma and rapid attainment of hemostasis. Other surgical options in this setting include intra-abdominal lavage and wide drainage for infected collections that are refractory to minimally invasive treatments either due to their size or the inspissated nature of their contents. Breakdown of a pancreaticojejunostomy may be salvaged in some situations by performing a pancreaticogastrostomy. ${ }^{117}$ In situations where there is complete dehiscence of the pancreaticoenterostomy and/or necrotizing pancreatitis, completion pancreatectomy may present the most appropriate and safe option for the patient, although this carries the very morbid sequela of brittle diabetes. ${ }^{118}$ Thus, surgical treatments are reserved for patients where minimally invasive methods are either inappropriate or have failed. ${ }^{119}$

\section{Supportive care}

The patient who has developed sepsis and/or hemorrhage as a result of POPF will also require supportive care. This includes management of the patient in the ICU, where appropriate inotropic and ventilatory support can be provided if necessary. The bleeding patient will also require judicious blood transfusion often with concomitant correction of coagulopathy especially in the setting of massive transfusion.

\section{Emerging concepts Pathophysiology/risk factors}

Challenging the notion that POPF is a factor that develops over time, work by de Reuver et $\mathrm{al}^{120}$ and Nahm et al ${ }^{121}$ demonstrates that high amylase concentration in immediate intraoperatively derived fluid from the peripancreatic environment (intraoperative amylase concentration, IOAC) is highly predictive of the development of POPF after PD and DP, respectively. IOAC was demonstrated to be an excellent predictor of POPF in both situations with an area under the receiver operator characteristic (AUROC) curve of 0.93 (95\% CI 0.87-0.99) for PD and 0.92 (95\% CI 0.81-0.99) in DP. These data suggested for the first time that the underlying pathophysiological events that lead to the eventual recognition of a POPF as per the ISGPF definition occur at the time of pancreatic resection.

The density of acinar cells at the pancreatic neck margin has also been demonstrated to correlate highly with the IOAC and the development of POP (as measured by urinary trypsinogen- 2 and serum amylase/lipase on postoperative day 1). The IOAC and the development of POP are, in turn, strongly associated with the development of POPF and a composite endpoint of systemic postoperative complications. This is supported by a growing body of biochemical evidence supporting the association between POP and POPF. ${ }^{122-124}$

The interaction between IOAC, acinar cell density, POP, and POPF has yet to be definitively elucidated; however, the authors have hypothesized a potential mechanism (Figure 1) whereby high-risk pancreata with a high acinar cell density are prone to both immediate leakage of protease-rich pancreatic fluid (IOAC) and the development of pancreatitis in the remnant gland as a result of ischemia and/or glandular manipulation.

The mechanism by which POP eventuates has yet to be explored in detail, although preliminary work by Ansorge et al 


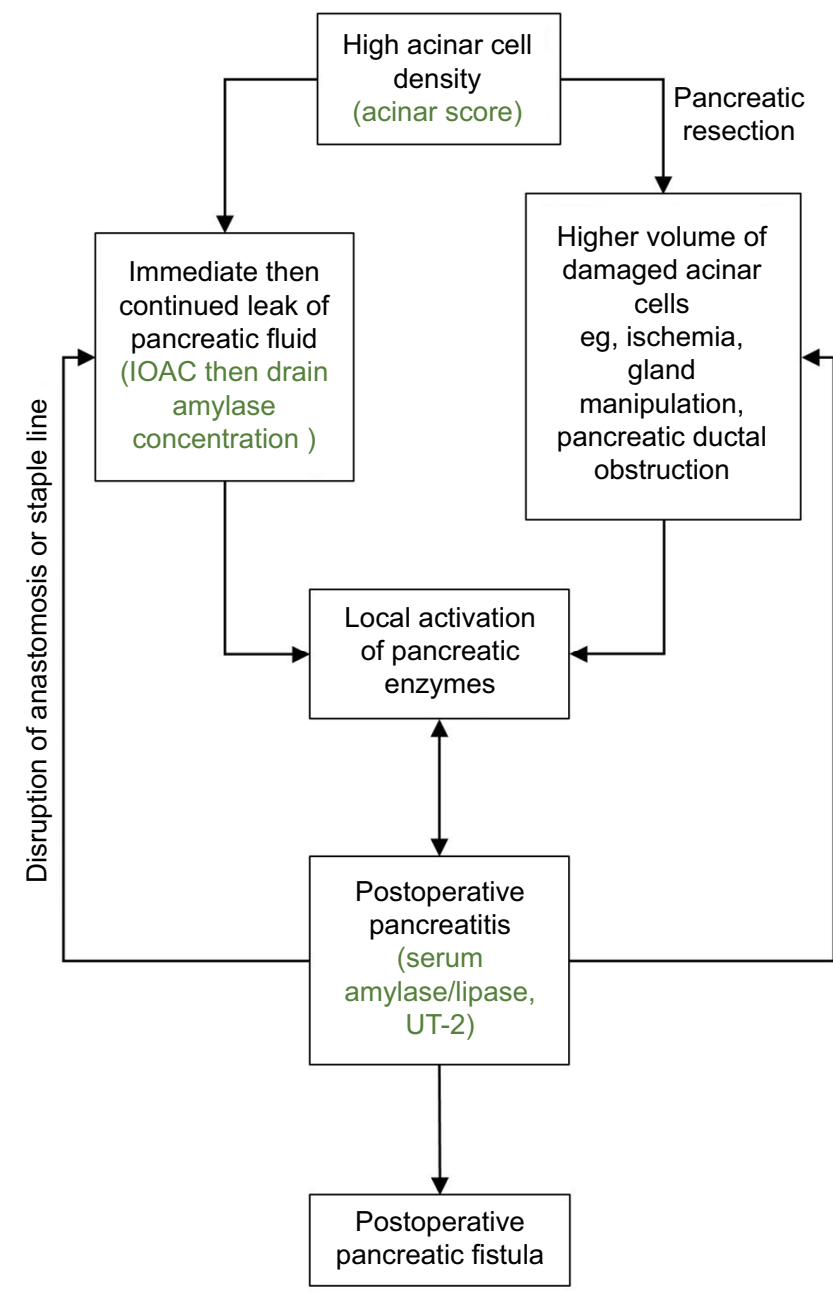

Figure I Hypothesized mechanism for the development of postoperative pancreatitis and POPF.

Abbreviations: IOAC, intraoperative amylase concentration; UT-2, urinary trypsinogen-2; POPF, postoperative pancreatic fistula.

suggests that focal ischemic pancreatitis may be involved. An observational cohort study of 48 patients undergoing PD was performed with measurement of plasma amylase concentration and intraperitoneal levels of lactate, pyruvate, and trypsinogen activation peptide (TAP) near the pancreatic anastomosis using microdialysis techniques. ${ }^{125}$ This revealed that there was a higher perianastomotic lactate/pyruvate ratio indicating local ischemia, and also significantly higher levels of perianastomotic TAP and plasma amylase, indicating that pancreatitis was associated with the development of POPF. This implicates pancreatitis as an important factor in the development of POPF, rather than a mere disruption of mechanical anastomotic integrity.

Further evidence of ischemia as a mechanism for POP includes the observations of altered blood flow following sharp division of the pancreatic neck during pancreatic resection. This has been observed directly by a lack of brisk bleeding, by Doppler assessment (lack of Doppler signal), ${ }^{126}$ and also using indocyanine green dye which demonstrates ischemia at the level of the pancreatic neck. ${ }^{127}$

The finding of POP as an integral step in the development of POPF brings significant implications for the prediction, prevention, and management of POPF.

\section{Prediction}

Biochemical markers of POP after pancreatic resection are evident from the first postoperative day. These include serum amylase and lipase, and urinary trypsinogen-2. In an observational study of 61 patients undergoing pancreatic resection, the presence of POP on the first postoperative day as determined by these markers was found to be a strong predictor of the development of POPF (OR 17.81, 95\% CI 2.17-145.9). ${ }^{128}$

Histological assessment of the pancreatic neck margin to determine the acinar cell density has also been shown to strongly predict the development of POPF (AUROC 0.744, $p=0.003) .{ }^{128}$ This may be determined in the intraoperative phase at the time of frozen section. Future studies in larger cohorts are required to validate this finding, and to assess whether the acinar cell density may be a more accurate and quantitative way to characterize the remnant gland than simply assessing glandular "texture."

\section{Prevention and management}

Studies investigating methods to mitigate the risk and severity of POPF should now not only include physical methods to reinforce or buttress the pancreatic anastomosis or staple line, but also include strategies to ameliorate POP. This may involve the prevention of pancreatic ischemia, reducing pancreatic inflammation, and agents to inhibit the activation of proteolytic enzymes. To date, the use of such strategies has not been evaluated in large-scale RCTs, and therefore cannot be recommended for routine clinical use. This includes the use of trypsin inhibitors, such as ulinastatin, which have been shown to decrease rates of both POP and POPF in two moderately sized RCTs. ${ }^{129,130}$ Moreover, a recent RCT evaluating intravenous hydrocortisone in 155 patients undergoing PD demonstrated a significant reduction in overall postoperative complications and a nonsignificant trend toward reduction in POPF. $^{131}$

\section{Conclusion}

Despite decades of research, rates of POPF have remained largely unchanged after pancreatic resection. This largely relates to a poor understanding of the pathophysiology of 
this complication. POP is now emerging as a possible critical factor in the underlying pathophysiology of POPF. Further research is required to investigate strategies to mitigate the effects of POP. Meanwhile, POPF remains a complex problem requiring a multidisciplinary approach to achieve effective prediction, prevention, and management.

\section{Disclosure}

The authors report no conflicts of interest in this work.

\section{References}

1. Iacono C, Verlato G, Ruzzenente A, et al. Systematic review of central pancreatectomy and meta-analysis of central versus distal pancreatectomy. Br J Surg. 2013;100(7):873-885.

2. Berger AC, Howard TJ, Kennedy EP, et al. Does type of pancreaticojejunostomy after pancreaticoduodenectomy decrease rate of pancreatic fistula? A randomized, prospective, dual-institution trial. $J \mathrm{Am}$ Coll Surg. 2009;208(5):738-747; discussion 747-749.

3. Pedrazzoli S. Pancreatoduodenectomy (PD) and postoperative pancreatic fistula (POPF): a systematic review and analysis of the POPFrelated mortality rate in 60,739 patients retrieved from the English literature published between 1990 and 2015. Medicine (Baltimore). 2017;96(19):e6858

4. Aston SJ, Longmire WP Jr. Management of the pancreas after pancreaticoduodenectomy. Ann Surg. 1974;179(3):322-327.

5. Bassi C, Dervenis C, Butturini G, et al. Postoperative pancreatic fistula: an international study group (ISGPF) definition. Surgery. 2005;138(1):8-13.

6. Bassi C, Marchegiani G, Dervenis C, et al. The 2016 update of the International Study Group (ISGPS) definition and grading of postoperative pancreatic fistula: 11 years after. Surgery. 2017;161(3):584-591.

7. Pratt WB, Maithel SK, Vanounou T, Huang ZS, Callery MP, Vollmer $\mathrm{CM}$ Jr. Clinical and economic validation of the International Study Group of Pancreatic Fistula (ISGPF) classification scheme. Ann Surg. 2007;245(3):443-451.

8. Daskalaki D, Butturini G, Molinari E, Crippa S, Pederzoli P, Bassi C. A grading system can predict clinical and economic outcomes of pancreatic fistula after pancreaticoduodenectomy: results in 755 consecutive patients. Langenbecks Arch Surg. 2011;396(1):91-98.

9. Cheng Y, Ye M, Xiong X, et al. Fibrin sealants for the prevention of postoperative pancreatic fistula following pancreatic surgery. Cochrane Database Syst Rev. 2016;2:CD009621.

10. Weniger M, D'Haese JG, Crispin A, Angele MK, Werner J, Hartwig W. Autologous but not fibrin sealant patches for stump coverage reduce clinically relevant pancreatic fistula in distal pancreatectomy: a systematic review and meta-analysis. World J Surg. 2016;40(11):2771-2781.

11. Yamamoto M, Hayashi MS, Nguyen NT, Nguyen TD, McCloud S, Imagawa DK. Use of Seamguard to prevent pancreatic leak following distal pancreatectomy. Arch Surg. 2009;144(10):894-899.

12. Buc E, Flamein R, Golffier C, et al. Peng's binding pancreaticojejunostomy after pancreaticoduodenectomy: a French prospective study. J Gastrointest Surg. 2010;14(4):705-710.

13. Jang JY, Chang YR, Kim SW, et al. Randomized multicentre trial comparing external and internal pancreatic stenting during pancreaticoduodenectomy. Br J Surg. 2016;103(6):668-675.

14. Aranha GV, Hodul P, Golts E, Oh D, Pickleman J, Creech S. A comparison of pancreaticogastrostomy and pancreaticojejunostomy following pancreaticoduodenectomy. J Gastrointest Surg. 2003;7(5):672-682.

15. Kitahata Y, Kawai M, Yamaue H. Clinical trials to reduce pancreatic fistula after pancreatic surgery - review of randomized controlled trials. Transl Gastroenterol Hepatol. 2016;1:4.

16. Callery MP, Pratt WB, Kent TS, Chaikof EL, Vollmer CM Jr. A prospectively validated clinical risk score accurately predicts pancreatic fistula after pancreatoduodenectomy. J Am Coll Surg. 2013;216(1):1-14.
17. Gaujoux S, Cortes A, Couvelard A, et al. Fatty pancreas and increased body mass index are risk factors of pancreatic fistula after pancreaticoduodenectomy. Surgery. 2010;148(1):15-23.

18. Han IW, Kim H, Heo J, et al. Excess intraoperative fluid volume administration is associated with pancreatic fistula after pancreaticoduodenectomy: a retrospective multicenter study. Medicine (Baltimore). 2017;96(22):e6893.

19. Kanda M, Fujii T, Suenaga M, et al. Estimated pancreatic parenchymal remnant volume accurately predicts clinically relevant pancreatic fistula after pancreatoduodenectomy. Surgery. 2014;156(3):601-610.

20. Kanda M, Fujii T, Kodera Y, Nagai S, Takeda S, Nakao A. Nutritional predictors of postoperative outcome in pancreatic cancer. Br J Surg. 2011;98(2):268-274

21. Kawai M, Kondo S, Yamaue H, et al. Predictive risk factors for clinically relevant pancreatic fistula analyzed in 1,239 patients with pancreaticoduodenectomy: multicenter data collection as a project study of pancreatic surgery by the Japanese Society of Hepato-Biliary-Pancreatic Surgery. J Hepatobiliary Pancreat Sci. 2011;18(4):601-608

22. Shamali A, De'Ath HD, Jaber B, et al. Elderly patients have similar short term outcomes and five-year survival compared to younger patients after pancreaticoduodenectomy. Int J Surg. 2017;45:138-143.

23. Wente MN, Veit JA, Bassi C, et al. Postpancreatectomy hemorrhage (PPH): an International Study Group of Pancreatic Surgery (ISGPS) definition. Surgery. 2007;142(1):20-25.

24. Aranha GV, Aaron JM, Shoup M, Pickleman J. Current management of pancreatic fistula after pancreaticoduodenectomy. Surgery. 2006;140(4):561-568; discussion 568-569.

25. Raty S, Sand J, Lantto E, Nordback I. Postoperative acute pancreatitis as a major determinant of postoperative delayed gastric emptying after pancreaticoduodenectomy. J Gastrointest Surg. 2006;10(8):1131-1139.

26. Morgan KA, Lancaster WP, Walters ML, et al. Enhanced recovery after surgery protocols are valuable in pancreas surgery patients. $\mathrm{JAm}$ Coll Surg. 2016;222(4):658-664.

27. Halle-Smith JM, Vinuela E, Brown RM, et al. A comparative study of risk factors for pancreatic fistula after pancreatoduodenectomy or distal pancreatectomy. HPB (Oxford). 2017;19(8):727-734.

28. Heeger K, Falconi M, Partelli S, et al. Increased rate of clinically relevant pancreatic fistula after deep enucleation of small pancreatic tumors. Langenbecks Arch Surg. 2014;399(3):315-321.

29. Falconi M, Mantovani W, Crippa S, Mascetta G, Salvia R, Pederzoli P. Pancreatic insufficiency after different resections for benign tumours. Br J Surg. 2008;95(1):85-91.

30. Watanabe H, Kanematsu M, Tanaka K, et al. Fibrosis and postoperative fistula of the pancreas: correlation with MR imaging findings - preliminary results. Radiology. 2014;270(3):791-799.

31. Kang JH, Park JS, Yu JS, et al. Prediction of pancreatic fistula after pancreatoduodenectomy by preoperative dynamic CT and fecal elastase-1 levels. PLoS One. 2017;12(5):e0177052.

32. Miller BC, Christein JD, Behrman SW, et al. A multi-institutional external validation of the fistula risk score for pancreatoduodenectomy. J Gastrointest Surg. 2014;18(1):172-179; discussion 179-180.

33. Ven Fong Z, Correa-Gallego C, Ferrone CR, et al. Early drain removalthe middle ground between the drain versus no drain debate in patients undergoing pancreaticoduodenectomy: a prospective validation study. Ann Surg. 2015;262(2):378-383.

34. Sato N, Tamura T, Minagawa N, Hirata K. Preoperative body mass index-to-prognostic nutritional index ratio predicts pancreatic fistula after pancreaticoduodenectomy. Hepatobiliary Surg Nutr. 2016;5(3):256-262.

35. Nishida Y, Kato Y, Kudo M, et al. Preoperative sarcopenia strongly influences the risk of postoperative pancreatic fistula formation after pancreaticoduodenectomy. J Gastrointest Surg. 2016;20(9):1586-1594.

36. Silver JK, Baima J. Cancer prehabilitation: an opportunity to decrease treatment-related morbidity, increase cancer treatment options, and improve physical and psychological health outcomes. Am J Phys Med Rehabil. 2013;92(8):715-727. 
37. Gerritsen A, Besselink MG, Gouma DJ, Steenhagen E, Borel Rinkes IH, Molenaar IQ. Systematic review of five feeding routes after pancreatoduodenectomy. Br J Surg. 2013;100(5):589-598; discussion 599.

38. Di Carlo V, Gianotti L, Balzano G, et al. Complications of pancreatic surgery and the role of perioperative nutrition. Dig Surg. 1999;16(4): 320-326.

39. Park JS, Chung HK, Hwang HK, Kim JK, Yoon DS. Postoperative nutritional effects of early enteral feeding compared with total parental nutrition in pancreaticoduodectomy patients: a prospective, randomized study. J Korean Med Sci. 2012;27(3):261-267.

40. Gianotti L, Braga M, Gentilini O, Balzano G, Zerbi A, Di Carlo V. Artificial nutrition after pancreaticoduodenectomy. Pancreas. 2000;21(4): 344-351.

41. Gerritsen A, Besselink MG, Cieslak KP, et al. Efficacy and complications of nasojejunal, jejunostomy and parenteral feeding after pancreaticoduodenectomy. J Gastrointest Surg. 2012;16(6):1144-1151.

42. Perinel J, Mariette C, Dousset B, et al. Early enteral versus total parenteral nutrition in patients undergoing pancreaticoduodenectomy: a randomized multicenter controlled trial (Nutri-DPC). Ann Surg. 2016;264(5):731-737.

43. Weimann A, Braga M, Harsanyi L, et al. ESPEN guidelines on enteral nutrition: surgery including organ transplantation. Clin Nutr. 2006;25(2):224-244.

44. Du L, Wang-Gillam A. Trends in neoadjuvant approaches in pancreatic cancer. J Natl Compr Canc Netw. 2017;15(8):1070-1077.

45. Cheng TY, Sheth K, White RR, et al. Effect of neoadjuvant chemoradiation on operative mortality and morbidity for pancreaticoduodenectomy. Ann Surg Oncol. 2006;13(1):66-74.

46. Takahashi H, Ogawa H, Ohigashi H, et al. Preoperative chemoradiation reduces the risk of pancreatic fistula after distal pancreatectomy for pancreatic adenocarcinoma. Surgery. 2011;150(3):547-556.

47. Ishikawa $\mathrm{O}$, Ohigashi $\mathrm{H}$, Imaoka $\mathrm{S}$, et al. Concomitant benefit of preoperative irradiation in preventing pancreas fistula formation after pancreatoduodenectomy. Arch Surg. 1991;126(7):885-889.

48. Heinrich S, Schafer M, Weber A, et al. Neoadjuvant chemotherapy generates a significant tumor response in resectable pancreatic cancer without increasing morbidity: results of a prospective phase II trial. Ann Surg. 2008;248(6):1014-1022.

49. Allen PJ, Gonen M, Brennan MF, et al. Pasireotide for postoperative pancreatic fistula. N Engl J Med. 2014;370(21):2014-2022.

50. Buchler M, Friess H, Klempa I, et al. Role of octreotide in the prevention of postoperative complications following pancreatic resection. $\mathrm{Am}$ J Surg. 1992;163(1):125-130; discussion 130-131.

51. Closset J, Journe S, Mboti F, El Nakadi I, Gelin M. Randomized controlled trial comparing somatostatin with octreotide in the prevention of complications after pancreatectomy. Hepatogastroenterology. 2008;55(86-87):1818-1823.

52. Fiess H, Klempa I, Hermanek P, et al. Prophylaxis of complications after pancreatic surgery: results of a multicenter trial in Germany. Digestion. 1994;55(Suppl 1):35-40.

53. Gouillat C, Chipponi J, Baulieux J, Partensky C, Saric J, Gayet B. Randomized controlled multicentre trial of somatostatin infusion after pancreaticoduodenectomy. Br J Surg. 2001;88(11):1456-1462.

54. Katsourakis A, Zezos P, Noussios G, et al. Does prophylactic administration of somatostatin decrease the rates of complications after pancreatic resection? A clinical and electron microscopy study. Pancreas. 2013;42(1):37-41.

55. Kollmar O, Moussavian MR, Richter S, de Roi P, Maurer CA, Schilling MK. Prophylactic octreotide and delayed gastric emptying after pancreaticoduodenectomy: results of a prospective randomized double-blinded placebo-controlled trial. Eur J Surg Oncol. 2008;34(8): 868-875.

56. Lange JR, Steinberg SM, Doherty GM, et al. A randomized, prospective trial of postoperative somatostatin analogue in patients with neuroendocrine tumors of the pancreas. Surgery. 1992;112(6):1033-1037; discussion 1037-1038.
57. Leandros E, Antonakis PT, Albanopoulos K, Dervenis C, Konstadoulakis MM. Somatostatin versus octreotide in the treatment of patients with gastrointestinal and pancreatic fistulas. Can J Gastroenterol. 2004;18(5):303-306.

58. Wang W, Tian B, Babu SR, Zhang Y, Yang M. Randomized, placebo-controlled study of the efficacy of preoperative somatostatin administration in the prevention of postoperative complications following pancreaticoduodenectomy. Hepatogastroenterology. 2013;60(123):400-405.

59. Yeo CJ, Cameron JL, Lillemoe KD, et al. Does prophylactic octreotide decrease the rates of pancreatic fistula and other complications after pancreaticoduodenectomy? Results of a prospective randomized placebo-controlled trial. Ann Surg. 2000;232(3):419-429.

60. Williams ST, Woltering EA, O'Dorisio TM, Fletcher WS. Effect of octreotide acetate on pancreatic exocrine function. Am J Surg. 1989;157(5):459-462.

61. Montorsi M, Zago M, Mosca F, et al. Efficacy of octreotide in the prevention of pancreatic fistula after elective pancreatic resections: a prospective, controlled, randomized clinical trial. Surgery. 1995;117(1):26-31.

62. Lowy AM, Lee JE, Pisters PW, et al. Prospective, randomized trial of octreotide to prevent pancreatic fistula after pancreaticoduodenectomy for malignant disease. Ann Surg. 1997;226(5):632-641.

63. Allen PJ. Pasireotide for postoperative pancreatic fistula. $N$ Engl J Med. 2014;371(9):875-876.

64. Ecker BL, McMillan MT, Asbun HJ, et al. Characterization and optimal management of high-risk pancreatic anastomoses during pancreatoduodenectomy. Ann Surg. Epub 2017 Jun 7.

65. Bruns H, Kortendieck V, Raab HR, Antolovic D. Intraoperative fluid excess is a risk factor for pancreatic fistula after partial pancreaticoduodenectomy. HPB Surg. 2016;2016:1601340.

66. Lavu H, Sell NM, Carter TI, et al. The HYSLAR trial: a prospective randomized controlled trial of the use of a restrictive fluid regimen with $3 \%$ hypertonic saline versus lactated Ringers in patients undergoing pancreaticoduodenectomy. Ann Surg. 2014;260(3):445-453; discussion 445-453.

67. Keck T, Wellner UF, Bahra M, et al. Pancreatogastrostomy versus pancreatojejunostomy for RECOnstruction after PANCreatoduodenectomy (RECOPANC, DRKS 00000767): perioperative and longterm results of a multicenter randomized controlled trial. Ann Surg. 2016;263(3):440-449.

68. Que W, Fang H, Yan B, et al. Pancreaticogastrostomy versus pancreaticojejunostomy after pancreaticoduodenectomy: a meta-analysis of randomized controlled trials. Am J Surg. 2015;209(6):1074-1082.

69. Borel F, Ouaissi M, Merdrignac A, et al. Pancreatico-jejunostomy decreases post-operative pancreatic fistula incidence and severity after central pancreatectomy. ANZ J Surg. Epub 2017 Aug 15.

70. Senda Y, Shimizu Y, Natsume S, et al. Randomized clinical trial of duct-to-mucosa versus invagination pancreaticojejunostomy after pancreatoduodenectomy. Br J Surg. 2018;105(1):48-57.

71. Singh AN, Pal S, Mangla V, et al. Pancreaticojejunostomy: does the technique matter? A randomized trial. J Surg Oncol. Epub 2017 Oct 16.

72. Bai X, Zhang Q, Gao S, et al. Duct-to-mucosa vs invagination for pancreaticojejunostomy after pancreaticoduodenectomy: a prospective, randomized controlled trial from a single surgeon. J Am Coll Surg. 2016;222(1):10-18.

73. Hua J, He Z, Qian D, Meng H, Zhou B, Song Z. Duct-to-mucosa versus invagination pancreaticojejunostomy following pancreaticoduodenectomy: a systematic review and meta-analysis. J Gastrointest Surg. 2015;19(10):1900-1909.

74. Sun X, Zhang Q, Zhang J, et al. Meta-analysis of invagination and duct-to-mucosa pancreaticojejunostomy after pancreaticoduodenectomy: an update. Int J Surg. 2016;36(Pt A):240-247.

75. Zhang S, Lan Z, Zhang J, et al. Duct-to-mucosa versus invagination pancreaticojejunostomy after pancreaticoduodenectomy: a metaanalysis. Oncotarget. 2017;8(28):46449-46460. 
76. Mauriello C, Polistena A, Gambardella C, et al. Pancreatic stump closure after pancreatoduodenectomy in elderly patients: a retrospective clinical study. Aging Clin Exp Res. 2017;29(Suppl 1):35-40.

77. Mezza T, Clemente G, Sorice GP, et al. Metabolic consequences of the occlusion of the main pancreatic duct with acrylic glue after pancreaticoduodenectomy. Am J Surg. 2015;210(4):783-789.

78. Suc B, Msika S, Fingerhut A, et al. Temporary fibrin glue occlusion of the main pancreatic duct in the prevention of intra-abdominal complications after pancreatic resection: prospective randomized trial. Ann Surg. 2003;237(1):57-65.

79. Orci LA, Oldani G, Berney T, et al. Systematic review and metaanalysis of fibrin sealants for patients undergoing pancreatic resection. $H P B$ (Oxford). 2014;16(1):3-11.

80. Wang Q, He XR, Tian JH, Yang KH. Pancreatic duct stents at pancreaticoduodenectomy: a meta-analysis. Dig Surg. 2013;30(4-6):415-424.

81. Winter JM, Cameron JL, Campbell KA, et al. Does pancreatic duct stenting decrease the rate of pancreatic fistula following pancreaticoduodenectomy? Results of a prospective randomized trial. J Gastrointest Surg. 2006;10(9):1280-1290; discussion 1290.

82. Motoi F, Egawa S, Rikiyama T, Katayose Y, Unno M. Randomized clinical trial of external stent drainage of the pancreatic duct to reduce postoperative pancreatic fistula after pancreaticojejunostomy. $\mathrm{Br} J$ Surg. 2012;99(4):524-531.

83. Pessaux P, Sauvanet A, Mariette C, et al. External pancreatic duct stent decreases pancreatic fistula rate after pancreaticoduodenectomy: prospective multicenter randomized trial. Ann Surg. 2011;253(5):879-885.

84. Poon RT, Fan ST, Lo CM, et al. External drainage of pancreatic duct with a stent to reduce leakage rate of pancreaticojejunostomy after pancreaticoduodenectomy: a prospective randomized trial. Ann Surg. 2007;246(3):425-433; discussion 433-435.

85. Dong Z, Xu J, Wang Z, Petrov MS. Stents for the prevention of pancreatic fistula following pancreaticoduodenectomy. Cochrane Database Syst Rev. 2016;5:CD008914.

86. Tani M, Kawai M, Hirono S, et al. A prospective randomized controlled trial of internal versus external drainage with pancreaticojejunostomy for pancreaticoduodenectomy. Am J Surg. 2010;199(6):759-764.

87. Diener MK, Seiler CM, Rossion I, et al. Efficacy of stapler versus handsewn closure after distal pancreatectomy (DISPACT): a randomised, controlled multicentre trial. Lancet. 2011;377(9776):1514-1522.

88. Bilimoria MM, Cormier JN, Mun Y, Lee JE, Evans DB, Pisters PW. Pancreatic leak after left pancreatectomy is reduced following main pancreatic duct ligation. Br J Surg. 2003;90(2):190-196.

89. Olah A, Issekutz A, Belagyi T, Hajdú N, Romics L Jr. Randomized clinical trial of techniques for closure of the pancreatic remnant following distal pancreatectomy. Br J Surg. 2009;96(6):602-607.

90. Uemura K, Satoi S, Motoi F, Kwon M, Unno M, Murakami Y. Randomized clinical trial of duct-to-mucosa pancreaticogastrostomy versus handsewn closure after distal pancreatectomy. Br J Surg. 2017;104(5):536-543.

91. Hamilton NA, Porembka MR, Johnston FM, et al. Mesh reinforcement of pancreatic transection decreases incidence of pancreatic occlusion failure for left pancreatectomy: a single-blinded, randomized controlled trial. Ann Surg. 2012;255(6):1037-1042.

92. Hayashibe A, Ogino N. Clinical study for pancreatic fistula after distal pancreatectomy with mesh reinforcement. Asian J Surg. Epub 2016 Dec 10.

93. Montorsi M, Zerbi A, Bassi C, Capussotti L, Coppola R, Sacchi M; Italian Tachosil Study Group. Efficacy of an absorbable fibrin sealant patch (TachoSil) after distal pancreatectomy: a multicenter, randomized, controlled trial. Ann Surg. 2012;256(5):853-859; discussion 859-860.

94. Tillmann LC, Herzog T, Uhl W, Belyaev O. Sealing with cyanoacrylate and a falciform patch cannot prevent postoperative pancreatic fistula. World J Surg. 2017;41(6):1601-1609.

95. Conlon KC, Labow D, Leung D, et al. Prospective randomized clinical trial of the value of intraperitoneal drainage after pancreatic resection. Ann Surg. 2001;234(4):487-493; discussion 493-494.
96. Van Buren G 2nd, Bloomston M, Hughes SJ, et al. A randomized prospective multicenter trial of pancreaticoduodenectomy with and without routine intraperitoneal drainage. Ann Surg. 2014;259(4):605-612.

97. Witzigmann H, Diener MK, Kienkotter S, et al. No need for routine drainage after pancreatic head resection: the dual-center, randomized, controlled PANDRA trial (ISRCTN04937707). Ann Surg. 2016;264(3):528-537.

98. Bassi C, Molinari E, Malleo G, et al. Early versus late drain removal after standard pancreatic resections: results of a prospective randomized trial. Ann Surg. 2010;252(2):207-214.

99. Shin SH, Kim SC, Song KB, et al. A comparative study of laparoscopic vs. open distal pancreatectomy for left-sided ductal adenocarcinoma: a propensity score-matched analysis. J Am Coll Surg. 2015;220(2): 177-185.

100. Bauman MD, Becerra DG, Kilbane EM, et al. Laparoscopic distal pancreatectomy for pancreatic cancer is safe and effective. Surg Endosc. 2018;32(1):53-61.

101. Xourafas D, Ashley SW, Clancy TE. Comparison of perioperative outcomes between open, laparoscopic, and robotic distal pancreatectomy: an analysis of 1815 patients from the ACS-NSQIP procedure-targeted pancreatectomy database. J Gastrointest Surg. 2017;21(9):1442-1452.

102. McMillan MT, Zureikat AH, Hogg ME, et al. A propensity scorematched analysis of robotic vs open pancreatoduodenectomy on incidence of pancreatic fistula. JAMA Surg. 2017;152(4):327-335.

103. Song KB, Kim SC, Hwang DW, et al. Matched case-control analysis comparing laparoscopic and open pylorus-preserving pancreaticoduodenectomy in patients with periampullary tumors. Ann Surg. 2015;262(1):146-155.

104. Klek S, Sierzega M, Turczynowski L, Szybinski P, Szczepanek K, Kulig J. Enteral and parenteral nutrition in the conservative treatment of pancreatic fistula: a randomized clinical trial. Gastroenterology. 2011;141(1):157-163.

105. Barnes SM, Kontny BG, Prinz RA. Somatostatin analog treatment of pancreatic fistulas. Int J Pancreatol. 1993;14(2):181-188.

106. Segal I, Parekh D, Lipschitz J, Gecelter G, Myburgh JA. Treatment of pancreatic ascites and external pancreatic fistulas with a long-acting somatostatin analogue (Sandostatin). Digestion. 1993;54(Suppl 1):53-58.

107. Gans SL, van Westreenen HL, Kiewiet JJ, Rauws EA, Gouma DJ, Boermeester MA. Systematic review and meta-analysis of somatostatin analogues for the treatment of pancreatic fistula. Br J Surg. 2012;99(6):754-760.

108. Smits FJ, van Santvoort HC, Besselink MG, et al. Management of severe pancreatic fistula after pancreatoduodenectomy. JAMA Surg. 2017;152(6):540-548.

109. Munoz-Bongrand N, Sauvanet A, Denys A, Sibert A, Vilgrain V, Belghiti J. Conservative management of pancreatic fistula after pancreaticoduodenectomy with pancreaticogastrostomy. J Am Coll Surg. 2004;199(2):198-203.

110. Halloran CM, Ghaneh P, Bosonnet L, Hartley MN, Sutton R, Neoptolemos JP. Complications of pancreatic cancer resection. Dig Surg. 2002;19(2):138-146.

111. Fabbri C, Luigiano C, Maimone A, Polifemo AM, Tarantino I, Cennamo V. Endoscopic ultrasound-guided drainage of pancreatic fluid collections. World J Gastrointest Endosc. 2012;4(11):479-488.

112. Tjaden C, Hinz U, Hassenpflug M, et al. Fluid collection after distal pancreatectomy: a frequent finding. HPB (Oxford). 2016;18(1):35-40.

113. Yekebas EF, Wolfram L, Cataldegirmen G, et al. Postpancreatectomy hemorrhage: diagnosis and treatment: an analysis in 1669 consecutive pancreatic resections. Ann Surg. 2007;246(2):269-280.

114. Schafer M, Heinrich S, Pfammatter T, Clavien PA. Management of delayed major visceral arterial bleeding after pancreatic surgery. HPB (Oxford). 2011;13(2):132-138.

115. Khalsa BS, Imagawa DK, Chen JI, Dermirjian AN, Yim DB, Findeiss LK. Evolution in the treatment of delayed postpancreatectomy hemorrhage: surgery to interventional radiology. Pancreas. 2015;44(6): 953-958. 
116. Bachellier P, Oussoultzoglou E, Rosso E, et al. Pancreatogastrostomy as a salvage procedure to treat severe postoperative pancreatic fistula after pancreatoduodenectomy. Arch Surg. 2008;143(10):966-970; discussion 971.

117. van Berge Henegouwen MI, De Wit LT, Van Gulik TM, Obertop H, Gouma DJ. Incidence, risk factors, and treatment of pancreatic leakage after pancreaticoduodenectomy: drainage versus resection of the pancreatic remnant. J Am Coll Surg. 1997;185(1):18-24.

118. Alexakis N, Sutton R, Neoptolemos JP. Surgical treatment of pancreatic fistula. Dig Surg. 2004;21(4):262-274.

119. de Reuver PR, Gundara J, Hugh TJ, Samra JS, Mittal A. Intra-operative amylase in peri-pancreatic fluid independently predicts for pancreatic fistula post pancreaticoduodectomy. HPB (Oxford). 2016;18(7):608-614.

120. Nahm CB, de Reuver PR, Hugh TJ, et al. Intra-operative amylase concentration in peri-pancreatic fluid predicts pancreatic fistula after distal pancreatectomy. J Gastrointest Surg. 2017;21(6):1031-1037.

121. Palani Velu LK, Chandrabalan VV, Jabbar S, et al. Serum amylase on the night of surgery predicts clinically significant pancreatic fistula after pancreaticoduodenectomy. HPB (Oxford). 2014;16(7):610-619.

122. Dalla Valle R, De Bellis M, Pedrazzi G, et al. Can early serum lipase measurement be routinely implemented to rule out clinically significant pancreatic fistula after pancreaticoduodenectomy? Int J Surg. 2015;21(Suppl 1):S50-S54.

123. Raty S, Sand J, Nordback I. Detection of postoperative pancreatitis after pancreatic surgery by urine trypsinogen strip test. Br J Surg. 2007;94(1):64-69.
124. Ansorge C, Regner S, Segersvard R, Strömmer L. Early intraperitoneal metabolic changes and protease activation as indicators of pancreatic fistula after pancreaticoduodenectomy. $\mathrm{Br} J$ Surg. 2012;99(1):104-111.

125. Strasberg SM, Drebin JA, Mokadam NA, et al. Prospective trial of a blood supply-based technique of pancreaticojejunostomy: effect on anastomotic failure in the Whipple procedure. J Am Coll Surg. 2002;194(6):746-758; discussion 759-760.

126. Subar D, Pietrasz D, Fuks D, Gayet B. A novel technique for reducing pancreatic fistulas after pancreaticojejunostomy. J Surg Case Rep. 2015;2015(7).

127. Nahm CB, Brown KM, Townend PJ, et al. Acinar cell density at the pancreatic resection margin is associated with post-pancreatectomy pancreatitis and the development of postoperative pancreatic fistula. HPB (Oxford). Epub 2018 Jan 5.

128. Uemura K, Murakami Y, Hayashidani Y, et al. Randomized clinical trial to assess the efficacy of ulinastatin for postoperative pancreatitis following pancreaticoduodenectomy. J Surg Oncol. 2008;98(5):309-313.

129. Zhang H, Tan C, Wang X, et al. Preventive effects of ulinastatin on complications related to pancreaticoduodenectomy: a consortprospective, randomized, double-blind, placebo-controlled trial. Medicine (Baltimore). 2016;95(24):e3731.

130. Laaninen M, Sand J, Nordback I, Vasama K, Laukkarinen J. Perioperative hydrocortisone reduces major complications after pancreaticoduodenectomy: a randomized controlled trial. Ann Surg. 2016;264(5):696-702.
Clinical and Experimental Gastroenterology

\section{Publish your work in this journal}

Clinical and Experimental Gastroenterology is an international, peerreviewed, open access, online journal publishing original research, reports, editorials, reviews and commentaries on all aspects of gastroenterology in the clinic and laboratory. This journal is included on PubMed. The manuscript management system is completely online

\section{Dovepress}

and includes a very quick and fair peer-review system, which is all easy to use. Visit http://www.dovepress.com/testimonials.php to read real quotes from published authors. 